\title{
Commentary: Auricular Neuromodulation: The Emerging Concept Beyond the Stimulation of Vagus and Trigeminal Nerves
}

Claire-Marie Rangon*

Head of Scientific Auriculotherapy Diploma, Faculty of Medicine, University of Paris-Saclay, 94276 Le Kremlin-Bicêtre, France

\section{Article Info}

\section{Article Notes}

Received: June 27, 2018

Accepted: July 23, 2018

\section{*Correspondence}

Dr Claire-Marie Rangon, Scientific Auriculotherapy Diploma, Département FMC, UFR Médecine Paris Sud, 63 rue Gabriel Péri, 94276 Le Kremlin-Bicêtre, France; Telephone No: +33 1 495967 96; E-mail: cmrangon@gmail.com.

(c) 2018 Rangon CM. This article is distributed under the terms of the Creative Commons Attribution 4.0 International License

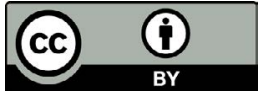

\section{Keywords}

Non-invasive neuromodulation Ear

Auricular neuromodulation

Vagus nerve; Trigeminal nerve

Superficial cervical plexus

Neurology
Abstract

The ears are a potential gateway to the brain, mainly through their innervation. Transcutaneous stimulation of the vagus (tVNS) and trigeminal (TNS) nerves at the auricles is gaining ground in the field of non-invasive brain therapeutics.

The concept of Auricular Neuromodulation (AN), described in the article " Auricular Neuromodulation: The emerging concept beyond the stimulation of vagus and trigeminal nerves" ${ }^{\prime 1}$ is particularly interesting for neurologists, and might even help researchers to unravel fundamental mechanisms of brain functioning.

\section{Introduction}

Non-invasive brain neuro-modulation is an exciting, growing field ${ }^{2-6}$. There are mainly three efficient techniques that are available: magnetic, ultrasound and electrical stimulations. Among non-invasive electrical stimulations, transcranial Electrical Stimulation (tES ), is the most common approach ${ }^{7}$. Nevertheless, electrical bottom-up techniques are making their way, in particular, transcutaneous Vagus Nerve Stimulation tVNS and Trigeminal Nerve Stimulation TNS ${ }^{6}$. Interestingly, these two techniques can be carried out by the stimulation of the external ear ${ }^{1}$. In other words, the auricles may be considered as a free gateway to the brain.

The Auricular Neuromodulation (AN) concept, developed in the article « Auricular Neuromodulation: The emerging concept beyond the stimulation of vagus and trigeminal nerves" ${ }^{\prime \prime}$, takes advantage of the three main nerve supplies of the ears, including the little-studied but promising superficial cervical plexus (SCP). This article aims to discuss why AN is particularly attractive in neurology, both for clinical and research purposes.

\section{Auricular Neuromodulation is Ideal for Clinical Neurology}

Among non-invasive techniques, there are at least three reasons to choose AN for treating chronic brain diseases. First, it is affordable. Second, it helps patients to stick to their treatments. Last, but not least, it takes advantage of a design by nature to reach the brain.

\section{An affordable non-invasive neuromodulation}

TNS and tVNS devices are easy to obtain by individuals via the world wide web. Moreover, they are affordable (a few hundred USD), depending on the brand, the number of electrodes, the quality of the 
battery, and the size of the device. Many people without health insurance can afford them. Therefore, AN should be considered by governments as an opportunity to improve health care access.

\section{An easy way to treat patients at home}

Moreover , AN is a very easy technique that is suitable for patient home care. It functions as a Transcutaneous Electrical Nerve Stimulation (TENS) device targeting the ear. It involves putting electrodes on the ears, selecting the right frequency, intensity, and duration of the electrical stimulation. As with TENS therapy, which has become very popular among chronic pain patients, we can bet AN will achieve similar success among neurological patients.

Certainly, AN is no longer the only non-invasive neuromodulation technique offering home treatment. Transcranial Direct Current Stimulation (tDCS) is becoming more available thanks to a recently developed "Adjustable Helmet Frame $»^{8}$. Nevertheless, the AN device is still more attractive because of its smaller size. Indeed, it complies with the anatomical shapes of the external ear. The tragus is easy to clip ${ }^{9}$ which makes this well-suited for everyday life. The latter is of capital importance since new AN devices can deliver continuous neurostimulation over several days while offering the patient a high degree of comfort and mobility (Primary Relief ${ }^{\mathrm{TM}}$ by DyAnsys ${ }^{\circledR}$ ). This advantage is particularly interesting when dealing with patients who have memory impairment or cognitive disorders ${ }^{10}$.

\section{A nature-designed technique to heal the brain through the guidance of neural plasticity and neuroimmunomodulation}

At last, as the ears are connected to the brain through their innervation, AN does not have to face either physical (i.e., the cranium) or chemical (i.e., the brain blood barrier) obstacles to treat brain disorders. The resulting stimulation is fast and brain-targeted.

A single electrical stimulation of the ear $(250 \mathrm{~Hz}, 0.14-$ $1.08 \mathrm{~mA}, 3 \mathrm{~V}, 4$ minutes) is able to directly modulate the activity of brain areas, thereby modifying significantly and specifically resting state EEGs ${ }^{11}$.

The vagus nerve, and consequently $\mathrm{AN}$, is also able to modulate the connectivity of brain areas; in other words, to guide neural plasticity ${ }^{12}$, as a « neural connectome $»^{13}$. For instance, tVNS significantly reduces symptoms in depressed patients ${ }^{14}$ and decreases also the functional connectivity between the bilateral medial hypothalamus and rostral anterior cingulate cortex ${ }^{15}$. In a stroke, tVNS favors not only neural plasticity by enhancing rehabilitation ${ }^{16,17}$, but also seems to strike at the root of brain lesions by decreasing the angiogenic response $\mathrm{e}^{18,19}$ and the infarct size $\mathrm{e}^{20,21}$.
Finally, the vagus nerve, and therefore AN, can stimulate indirectly the brain, via the modulation of the immune system $^{22,23}$. In epilepsy, a common neuroinflammatory disease, a randomized double-blind study including 76 drug-resistant patients ${ }^{24}$ showed a significant reduction of seizures after 20 weeks of tVNS $(25 \mathrm{~Hz}, 4$ hours daily; $\mathrm{p}=0.034$ ). Ear electrostimulation of kainic acid-induced epileptic rats confirmed tVNS efficacy against brain inflammatory mediators ${ }^{25,26}$.

Thus, AN is a promising method that is consistent with several expectations of neurologists. Neuroscientists as well might soon get interested in AN.

\section{Auricular Neuromodulation is Promising for Brain Research}

\section{Unravelling brain hubs through mass ear recordings}

An excellent review, published in April 2018, suggests that «non-invasive neuromodulation affects a brain network rather than just the local stimulation site targeted $»^{27}$. In humans, the identification of the major brain hubs requires time and money to collect and compare using structural and functional neuroimaging studies ${ }^{28,29}$. No matter how many hundreds or thousands of neuroimaging studies are available in the data banks, it virtually impossible to get an exhaustive data collection that takes into account all the neurodevelopmental stages, all the genetic backgrounds, even all the epigenetic disturbances.

In contrast, electrical resistance recordings of the ears are much easier to get from millions of people than from functional neuroimaging studies, provided that the recording time is not too long, the procedure is accurate, painless, affordable and ideally easy to move. Besides, significant changes of the electrical resistance recorded on the auricules may also serve as early screening tests for specific brain diseases. For instance, for the early diagnosis of dementia ${ }^{30,31}$, auricular electrical resistance measurements would be much easier to carry out than vagus somatosensory potentials recordings.

Fortunately, this kind of device is already available! It is currently used by auricular acupuncturists to detect active acupoints. Interestingly, in an Austrian study, the ears of neonates suffering from neonatal abstinence syndrome were screened : one hundred percent of the neonates presented active acupoints, including in the ear area supplied by the $\mathrm{SCP}^{32}$. In addition, another auricular acupuncture randomized trial studying the efficacy of selected ear acupoints in migraine attacks concluded that the point located in the area supplied by the SCP was significantly more efficient than another point located above the concha ${ }^{33}$. This result was not surprising because, in auriculotherapy maps, the head is always represented within the earlobe, supplied by the superficial cervical 
plexus («the inverted fetus »). Furthermore, other clinical applications of auriculotherapy in neurology have been cited lately and provide an evidence-based medicine overview of this still little-known field of research ${ }^{34}$.

Likewise, up and coming basic neuroscience research should also pay attention to the detection and stimulation of the ear area supplied by the superficial cervical plexus. Optogenetics manipulations may turn out to be the best option to validate the hypothesis of the auricular brain map in animal models. Indeed, optogenetics, a revolutionary research tool based upon bioengineered light-sensitive proteins, can optionally stimulate or silence particular cell types and neuronal circuits with milliseconds temporal accuracy $^{35-37}$. Therefore, optogenetics might represent an alternative cutting edge approach in term of Auricular Neuromodulation both for the validation of the "ear hubs" corresponding to the brain hubs, potentially through transdermal ear illumination ${ }^{38}$ and for unravelling the neural circuits involved in ear stimulation ${ }^{39,40}$. Finally, optogenetic manipulations may also contribute to optimize the ear stimulation parameters ${ }^{41}$.

\section{Optimizing ear stimulation parameters}

Aside from defining the ear points, the optimal characteristics of the electrical stimulation have to be precisely determined. First, the frequency is of capital importance, otherwise, the stimulation even of the right ear point will not trigger any significant improvement ${ }^{42,24}$. Along these lines, the intensity of the optimal current, the wavelength and the duration of the ear stimulation each require scientific evaluation.

Once more, sharing the experience of ear acupuncturists should help neuroscientists. For instance, semi-permanent needles are inserted into the ear according to the electrical charge of the acupoint (thereby indicating whether the acupoint is active or not), which is a technique that gives a sense of the scale of the magnitude of the necessary stimulation to apply. Laser stimulation parameters, applied to the ears of neonates, could also help researchers get a headstar $t^{43}$.

\section{Conclusion}

Auricular Neuromodulation is only in its early stages. So far, studies have concerned mainly tVNS. In view of the tremendous potential of the AN as an amazing concept, leading neuroscience research laboratories as well as other interested decision-makers should join in and show the way.

\section{Conflict of interest statement}

The author has no conflicts of interest.

\section{Funding source}

The Faculty of Medicine of the University of Paris-Saclay supported the publication of this article.

\section{Abbreviations}

AN: Auricular Neuromodulation

SCP: Superficial Cervical Plexus

tDCS: transcranial Direct Current Stimulation

tES: transcranial Electrical Stimulation

TNS: Trigeminal Nerve Stimulation

tVNS: transcutaneous Vagus Nerve Stimulation

\section{References}

1. Mercante B, Deriu F, Rangon CM. Auricular Neuromodulation: The Emerging Concept beyond the Stimulation of Vagus and Trigeminal Nerves. Medicines (Basel). 2018; 5.

2. Krishna V, Sammartino F, Rezai A. A Review of the Current Therapies, Challenges, and Future Directions of Transcranial Focused Ultrasound Technology: Advances in Diagnosis and Treatment. JAMA Neurol. 2018; 75: 246-54.

3. Palm U, Kumpf U, Behler N, et al. Home Use, Remotely Supervised, and Remotely Controlled Transcranial Direct Current Stimulation: A Systematic Review of the Available Evidence. Neuromodulation. 2017.

4. Tavakoli AV, Yun K. Transcranial Alternating Current Stimulation (tACS) Mechanisms and Protocols. Front Cell Neurosci. 2017; 11: 214.

5. Goetz SM, Deng ZD. The development and modelling of devices and paradigms for transcranial magnetic stimulation. Int Rev Psychiatry. 2017; 29: 115-45.

6. Shiozawa P, Silva ME, Carvalho TC, et al. Transcutaneous vagus and trigeminal nerve stimulation for neuropsychiatric disorders: a systematic review. Arq Neuropsiquiatr. 2014; 72: 542-7.

7. Bucur M, Papagno C. A systematic review of noninvasive brain stimulation for post-stroke depression. J Affect Disord. 2018; 238: 69-78.

8. Cancelli A, Cottone C, Giordani A, et al. MRI-Guided Regional Personalized Electrical Stimulation in Multisession and Home Treatments. Front Neurosci. 2018; 12: 284.

9. Badran BW, Brown JC, Dowdle LT, et al. Tragus or cymba conchae? Investigating the anatomical foundation of transcutaneous auricular vagus nerve stimulation (taVNS). Brain Stimul. 2018; 11: 947-48.

10. Devinsky O, Asato M, Camfield P, et al. Delivery of epilepsy care to adults with intellectual and developmental disabilities. Neurology. 2015; 85: 1512-21.

11. Mielczarek M, Michalska J, Polatynska K, et al. An Increase in Alpha Band Frequency in Resting State EEG after Electrical Stimulation of the Ear in Tinnitus Patients-A Pilot Study. Front Neurosci. 2016; 10: 453.

12. Hays SA. Enhancing Rehabilitative Therapies with Vagus Nerve Stimulation. Neurotherapeutics. 2016; 13: 382-94.

13. Edgerton VR, Gad P. Is the vagus nerve our neural connectome. Elife. $2018 ; 7$.

14. Kong J, Fang J, Park J, et al. Treating Depression with Transcutaneous Auricular Vagus Nerve Stimulation: State of the Art and Future Perspectives. Front Psychiatry. 2018; 9: 20.

15. Tu Y, Fang J, Cao J, et al. A distinct biomarker of continuous transcutaneous vagus nerve stimulation treatment in major depressive disorder. Brain Stimul. 2018; 11: 501-08.

16. Redgrave JN, Moore L, Oyekunle T, et al. Transcutaneous Auricular Vagus Nerve Stimulation with Concurrent Upper Limb Repetitive 
Task Practice for Poststroke Motor Recovery: A Pilot Study. J Stroke Cerebrovasc Dis. 2018; 27: 1998-2005.

17. Capone F, Miccinilli S, Pellegrino G, et al. Transcutaneous Vagus Nerve Stimulation Combined with Robotic Rehabilitation Improves Upper Limb Function after Stroke. Neural Plast. 2017; 2017: 7876507.

18. Jiang Y, Li L, Ma J, et al. Auricular vagus nerve stimulation promotes functional recovery and enhances the post-ischemic angiogenic response in an ischemia/reperfusion rat model. Neurochem Int. 2016; 97: 73-82.

19. Ma J, Zhang L, He G, et al. Transcutaneous auricular vagus nerve stimulation regulates expression of growth differentiation factor 11 and activin-like kinase 5 in cerebral ischemia/reperfusion rats. J Neurol Sci. 2016; 369: 27-35.

20. Yang Y, Yang LY, Orban L, et al. Non-invasive vagus nerve stimulation reduces blood-brain barrier disruption in a rat model of ischemic stroke. Brain Stimul. 2018; 11: 689-98.

21. Ay I, Napadow V, Ay H. Electrical stimulation of the vagus nerve dermatome in the external ear is protective in rat cerebral ischemia. Brain Stimul. 2015; 8: 7-12.

22. Pavlov VA, Chavan SS, Tracey KJ. Molecular and Functional Neuroscience in Immunity. Annu Rev Immunol. 2018; 36: 783-812.

23. Han B, Li X, Hao J. The cholinergic anti-inflammatory pathway: An innovative treatment strategy for neurological diseases. Neurosci Biobehav Rev. 2017; 77: 358-68.

24. Bauer S, Baier H, Baumgartner C, et al. Transcutaneous Vagus Nerve Stimulation (tVNS) for Treatment of Drug-Resistant Epilepsy: A Randomized, Double-Blind Clinical Trial (cMPsE02). Brain Stimul. 2016; 9: 356-63.

25. Liao ET, Lin YW, Huang CP, et al. Electric Stimulation of Ear Reduces the Effect of Toll-Like Receptor 4 Signaling Pathway on Kainic AcidInduced Epileptic Seizures in Rats. Biomed Res Int. 2018; 2018: 5407256.

26. Liao ET, Tang NY, Lin YW, et al. Long-term electrical stimulation at ear and electro-acupuncture at ST36-ST37 attenuated COX-2 in the CA1 of hippocampus in kainic acid-induced epileptic seizure rats. Sci Rep. $2017 ; 7: 472$

27. To WT, De Ridder D, Hart J, Jr., Vanneste S. Changing Brain Networks Through Non-invasive Neuromodulation. Front Hum Neurosci. 2018; 12: 128 .

28. Goodkind M, Eickhoff SB, Oathes DJ, et al. Identification of a common neurobiological substrate for mental illness. JAMA Psychiatry. 2015; 72: 305-15.

29. Downar J, Blumberger DM, Daskalakis ZJ. The Neural Crossroads of
Psychiatric Illness: An Emerging Target for Brain Stimulation. Trends Cogn Sci. 2016; 20: 107-20.

30. Polak T, Herrmann MJ, Muller LD, et al. Near-infrared spectroscopy (NIRS) and vagus somatosensory evoked potentials (VSEP) in the early diagnosis of Alzheimer's disease: rationale, design, methods, and first baseline data of the Vogel study. J Neural Transm (Vienna). 2017; 124: 1473-88.

31. Polak T, Ehlis AC, Langer JB, et al. Non-invasive measurement of vagus activity in the brainstem - a methodological progress towards earlier diagnosis of dementias. J Neural Transm (Vienna). 2007; 114: 613-9.

32. Raith W, Kutschera J, Muller W, et al. Active ear acupuncture points in neonates with neonatal abstinence syndrome (NAS). Am J Chin Med. 2011; 39: 29-37.

33. Allais G, Romoli M, Rolando S, et al. Ear acupuncture in the treatment of migraine attacks: a randomized trial on the efficacy of appropriate versus inappropriate acupoints. Neurol Sci. 2011; 32 Suppl 1: S173-5.

34. Stanton G. Auriculotherapy in Neurology as an Evidence-Based Medicine: A Brief Overview. Med Acupunct. 2018; 30: 130-32.

35. Mahmoudi P, Veladi H, Pakdel FG. Optogenetics Tools and Applications in Neurobiology. J Med Signals Sens. 2017; 7: 71-79.

36. Liu S, Li C, Xing Y, et al. Role of Neuromodulation and Optogenetic Manipulation in Pain Treatment. Curr Neuropharmacol. 2016; 14 . 654-61.

37. Boyden ES. Optogenetics and the future of neuroscience. Nat Neurosci. $2015 ; 18: 1200-1$.

38. Maimon BE, Zorzos AN, Bendell R, et al. Transdermal optogenetic peripheral nerve stimulation. J Neural Eng. 2017; 14: 034002.

39. Jhang J, Lee H, Kang MS, et al. Anterior cingulate cortex and its input to the basolateral amygdala control innate fear response. Nat Commun. 2018; 9: 2744.

40. Shin SS, Pelled G. Novel Neuromodulation Techniques to Assess Interhemispheric Communication in Neural Injury and Neurodegenerative Diseases. Front Neural Circuits. 2017; 11: 15.

41. Edward ES, Kouzani AZ, Tye SJ. Towards miniaturized closed-loop optogenetic stimulation devices. J Neural Eng. 2018; 15: 021002.

42. Wang Z, FangJ, Liu J, etal. Frequency-dependent functional connectivity of the nucleus accumbens during continuous transcutaneous vagus nerve stimulation in major depressive disorder. J Psychiatr Res. 2018; 102: 123-31.

43. Raith W, Schmolzer GM, Resch B, et al. Laser Acupuncture for Neonatal Abstinence Syndrome: A Randomized Controlled Trial. Pediatrics. 2015; 136 : 876-84. 